\title{
Research on Virtual Experiment Environment Construction and Teaching Practice Based on Web3D Technology
}

\author{
Yan Li and Liping Wang \\ ${ }^{1}$ Pingxiang University, Pingxiang, Jiangxi 337000
}

\begin{abstract}
Keywords: Virtual Experiment Environment; Web3D Technology; Teaching Practice
\end{abstract}
\begin{abstract}
The virtual experiment environment is a kind of software and hardware operating environment that is built on the computer by means of graphics, simulation and virtual reality technologies to assist or replace the traditional experimental operations. The virtual experiment provides a good solution for the experimental teaching in the gradually developing network education with its own characteristics. This paper first analyzes the principles and methods followed by the construction of virtual experiment environment, then introduces the advantages of Web3D technology, compares the four Web3D technologies, and finally lists the application of Web3D technology in teaching practice.
\end{abstract}

\section{Introduction}

A large number of new computer-based technologies are used in education and teaching. These technologies can greatly help teaching from the aspects of teaching resources, teaching process, teaching evaluation, and teaching effects. Web3D technology is currently using relatively new computer virtual reality technology. Therefore, it is very meaningful to study the application of Web3D technology in teaching.[1] Web3D is a technology that implements virtual reality technology. It refers to the Internet-based, desktop-level virtual reality technology that is implemented by software technology. The earliest development of Web3D can be traced back to VRML (Virtual Reality Modeling Language). In the spring of 2000, Web3D completed the conversion from VRML to X-3D. The X-3D standard integrates the rapidly developing XML, advanced technologies such as JAVA and streaming media provide Web3D with more powerful and efficient 3D computing capabilities, rendering quality and transmission speed, and today, Web3D technology is increasingly used in merchandise display, process simulation, urban planning and entertainment. The same technology has also been applied to education and teaching, and has become an important teaching technology.Web3D can play an important role in the virtual teaching environment, and the teaching environment needs the function of contextualization and natural interaction. It can break the limitation of time and space, overcome the shortcomings of various realistic teaching conditions, help students better understand and study the teaching content, and can also use Web3D technology to conduct virtual laboratory work, improve the students' experimental skills, and save experimental supplies. [2]

\section{The Principle of Constructing Virtual Experiment Environment}

With the continuous development of artificial intelligence technology, its application is in all aspects of education and teaching, and virtual experiments will be no exception. The intelligentization of virtual experiments will play a greater role in distance education[2].

The principle of consistency

The principle of consistency is the most basic principle in the design and development of virtual experiments, that is, it must be consistent with the nature of things in real experiments, and must be consistent with the students' cognitive laws. The network virtual experiment must strictly abide by this principle in the process of design development, otherwise it will give students wrong understanding in the application of remote experiment teaching.

Openness principle. Openness is an important principle of network virtual experiments, and it is also the purpose of designing virtual experiments on the Internet. It can provide students with 
maximum freedom and flexibility. Virtual experiments can only meet the diversified needs of experimenters only when they are completely open in time, space, content, and organizational form, so as to better solve the experimental problems in network teaching.

The interaction principle. If there is no interaction, the understanding of the virtual environment can only be determined in advance by the system. Observing along a specific path, the experiment will only play a demonstration role. The difference is too big. The interaction of virtual experiments enables students to simulate the actual experimental operation process. Students can improve practical skills by doing virtual experiments. Students can exchange learning experiences, experimental methods and experiments on the Internet through various interactive means provided by the virtual experiment system.

Redundancy principle. Redundancy does not mean simple repetition, but refers to different expressions of the same information content. Psychological studies have shown that the appearance of the same information in different forms and at different times can enhance students' memory and understanding of their content. In the design of virtual experiments, various forms of expression such as charts, images, and speech can be fully considered so that students can achieve good memory effects.

Combination criteria. The virtual experiment system not only involves learners, but also includes developers, service providers and others. In the system design and development, it is necessary to rationally divide experimental content and programming techniques, consider a variety of computer platforms and user interface standards, programming and component communication standards, establish a unified design specification, in the future development of new functions and equipment out .When updating, it can be more convenient to improve the performance of the system.

Intuitive principle. The virtual experiment system needs a personal and friendly interface in the design process, otherwise it will make students feel a sense of resistance. The presentation of experimental data, experimental results, and experimental phenomena should be obvious and intuitive, and it is easy for students to feel the changes that occur during the experimental operation at the first time, and they cannot be like hide-and-seek. In addition to the design principles of some of the network virtual experiments mentioned above, we also need to consider the security of virtual experimental systems, and the ease of management and maintenance of daily virtual experiment systems and the speed with which virtual experiments run on the network.

\section{The Method to Build a Virtual Experiment Environment}

Virtual experiment systems usually contain the following important components:

(1) a server that can handle a large number of analog operation data processing (such as a national computing center, a high-performance computer system on campus, or a network computing system of a company's R\&D department);

(2) a database system for storing initial conditions and boundary conditions of simulation data and experimental result data;

(3) scientific experimental equipment and cooperation tools connected to the Internet;

(4)the experimental processing software, each virtual experimental system is based on professional simulation software system, they are used for data processing, analysis, storage and visualization of this process. [3]

The close integration of these parts forms a virtual experimental system [2]. The virtual experiment system discussed in this article is based on Web3D technology. The Web3D technology is mainly used and embodied in the client. In order to reduce the burden on the client, the software environment of the client is required to be a browser only, and the hardware and network conditions should also be suitable for the current medium configuration. To visualize a $3 \mathrm{D}$ environment in a web browser, each visual 3D object represents an experimental object. The user can perform virtual experiments by clicking and dragging the mouse. 


\section{The Different Kinds of Web3D Technologies}

Web3 D can be seen simply as a combination of Web technology and 3D technology. It is actually the extension of the native 3D graphics technology to the Internet. Its essential characteristics are network, three-dimensional, and interactivity[3].

Cult3D. Cult3D is a brand new technology launched by Cycore of Sweden. Its basic idea is to use existing network technology and powerful 3D engine to create interactive 3D objects on web pages. The core of Cult3 D is based on Java. It can also be embedded in Java classes developed by customers themselves, so it has strong interaction and expansion performance. Cult 3 D consists of 3 different program functions - Cult3D Exporter plug-in, Cult3D Designer and Cult3D Viewer plug-in. Web developers can design 3D models using 3DSMAX or MAYA widely used in the 3D design field. , Use Cult3D Exporter plug-in to convert the design model, add other effects such as interaction and sound effects to the model in Cult3D Designer, and then seamlessly embed it into HTML pages and other applications. Users only need to install Cult3D Viewer plug-in. The 3D model generated using Cult3D technology can be viewed on the Internet in real time, and can be interactively rotated, enlarged or reduced by the mouse. From this development process, we can see that developers do not need to adapt to new technologies, and Cult3D can provide users with humanized and organized development interfaces. The operation is simple and intuitive, which greatly improves the development efficiency and reduces the cost of end users. Cult3D is a cross-platform 3D rendering engine that delivers real-time interactive objects with unprecedented quality and speed to all Internet users.

Viewpoint. Viewpoint is a Web3D solution proposed by the United States Viewpoint Corporation. Using its XML-based language framework can be easily communicated with the browser and database, but also can be embedded in a number of software, which makes Viewpoint's application extensive. The user only needs to install a simple and free plug-in to view the 3D model transmitted in streaming mode on the Internet. At the same time, the user can also control the rotation, zoom, etc. The focus of the technology covers all aspects of Web3D, unlike other solutions. Viewpoint creates, processes, and transmits 3D graphics objects itself instead of importing 3D images from other sources. Viewpoint can be divided into two parts in its structure. One is a mts file for storing 3D data and texture data, and the other is an XML-based mtx file for describing scene parameters and interactions. The file format generated by Viewpoint is very small, plus its 3D polygonal mesh structure is scalable. And streaming, which makes it ideal for transmission over the network. Scalability means the object can automatically adjust the accuracy and other related parameters according to the processing speed of the user's machine and the speed of the modem to obtain the best performance. In this way, the designer can develop a high-resolution model to meet the user's different levels of accuracy. Needed to reduce development time. Streaming allows users to see the model content without having to download all the 3D data, and even interact with $3 \mathrm{D}$ objects during the download process. It uses proprietary compression techniques to compress complex 3D information into a very small number format, while also ensuring that our browser plugins can quickly compress these The information is reinterpreted. Its compression ratio is higher than any existing 3D compression technology on the Internet. Its extremely small file size enables users to browse Viewpoint format objects on the Internet more quickly. In terms of 3D mapping, it uses the JPEG compression format to ensure that the file's texture does not make the 3D file larger. It also has a pure software quality. The real-time rendering engine, which does not require any hardware acceleration device, has a rendering effect close to that of the real world. The main application market of Viewpoint is the product promotion and e-commerce field as an item display. Currently Viewpoint has provided solutions for e-commerce and online advertising to famous companies such as Nike, Sony, and Sharper Image[3].

Atmosphere. Atmosphere is an authoritative position in the field of image processing and publishing. Adobe launched a three-dimensional virtual environment-based online chat tool that can connect multiple users via the Internet. It integrates the creation, viewing, and interaction of a virtual 3D world. Professional development solution for the virtual 3D environment. Although the software is still in the debug development stage, due to its unique light energy tracking algorithm, it 
has great advantages in the display of indoor space. It deserves our attention. Adobe Atmosphere contains 3 Components: Atmosphere Builder, which provides excellent 3D modeling techniques to build 3D virtual worlds; Atmosphere Browser, a free browser plug-in provided by Adobe (can also be run as a stand-alone application), enabling users to browse Adobe Atmosphere The 3D world created, the browser interface; Atmosphere Community Server, enables users to use a text-based interface to communicate with other users in the 3D world created by Adobe Atmosphere. Atmosphere's browser operation interface needs attention. Yes, Atmosphere uses view-point Technology to achieve high-quality compression and real-time rendering of 3D geometry, so installing the Atmosphere browser plug-in also installed the Viewpoint plug-in. Atmosphere in addition to providing their own modeling tools Atmosphere Builder to create a three-dimensional virtual world, 3D models created by 3D authoring tools such as 3DSMAX or Lightwave can be exported to Viewpoint format files (*.mts and *.mtx files) and imported into Atmosphere Builder. At the same time, Atmosphere can also perform well. Compatible with industry-standard web content design tools such as Adobe Photo-shop and Adobe Illustrator, it can also incorporate standard web image formats into Atmosphere such as JPEG, GIF, and PNG. Adobe Atmosphere provides us with an excellent virtual community solution, but because it is in the debug development stage, there are still some inadequacies: From the perspective of modeling technology, the quality of the generated scene is still relatively rough; from the perspective of rendering technology. The size of the Atmosphere Browser browser (5MB) is too large for the dial-up users to download time; from the perspective of short message chat, only support one-to-many; only from the perspective of scalability.[3]The browser and its own player do not support embedding in other environments. From the perspective of server-side support, Adobe has not yet provided a server-side program to handle multi-user interactive messaging. The current Atmosphere scenario is only available. Connected to Adobe's server; from the operating platform point of view, the current Beta version can only be installed on the Windows operating platform, only the official version can be used for both Windows and Mac platforms.

Shout3D. Shout3 D is Shout Interactive solution for transferring interactive 3D graphics and animations over the Internet. Designers can use 3DS MAX or other modeling tools to create basic models (including modeling, textures, materials, lighting, and (Cameras, etc.) The Shout3D plugin for 3DS MAX outputs the model directly to Shout3D's .s3d file format (other modeling tools output it as a wrl file format) and can then use existing applets, or According to their own needs, use Java or Java Script to develop any imaginable interactive capabilities, and finally use the Shout3 D Wizard to publish interactive 3D animations to the Internet. Although Shout3 D generates a low-quality scene, it uses it to provide High interactivity can easily develop 3D games on the Internet. Figure 4 shows an example of such a game. Shout3D is a $3 \mathrm{D}$ graphics rendering engine based on Java applet, and it is also a production tool for Web3 D graphics. Because it is used Java technology to deliver interactive 3D graphics on the web, when visitors browse pages with Shout3D, its content and player Downloaded at the same time. And this player is embedded in the web applet program, we know that all versions of Microsoft IE and Netscape4.0 and above can run Java virtual machine, so users do not have to worry about operating platform limitations. 
Table 1 Comparison of 4 Web3D Technologies

\begin{tabular}{|l|l|l|l|}
\hline Forms & Plug-in & Software & Advantages \\
\hline Cult3D & requires plug-in & Win/Mac OS & $\begin{array}{l}\text { Based on Java,small file size, good } \\
\text { image quality, no hardware support, can } \\
\text { be used for Office or Acrobat documents }\end{array}$ \\
\hline Viewpoint & requires plug-ins & Win/Mac & $\begin{array}{l}\text { Scalable, streaming, high compression } \\
\text { ratio }\end{array}$ \\
\hline Shout3D & requires plug-in & $\begin{array}{l}\text { Beta : Win } \\
\text { Official version : } \\
\text { Win/Mac OS }\end{array}$ & $\begin{array}{l}\text { Connect multiple users to achieve virtual } \\
\text { community, light tracking algorithm } \\
\text { indoor display effect is good, natural } \\
\text { gravity and collision simulation }\end{array}$ \\
\hline & $\begin{array}{l}\text { does not require } \\
\text { plug-in }\end{array}$ & $\begin{array}{l}\text { Win/ Unix/ Mac } \\
\text { OS }\end{array}$ & $\begin{array}{l}\text { Based on Java Applet, follow X3D } \\
\text { specification }\end{array}$ \\
\hline
\end{tabular}

\section{The Advantages of Wed3D Technologies}

Learning takes place in the interaction between the individual and the external environment. The learner's personal experience in real or virtual reproduction is far more profound than the indirect experience represented by other people's descriptions and symbols, and is more conducive to the construction of meaning. . The significant advantage of Web3d is its ability to provide a highly interactive learning environment. It is a learning scenario tool that can provide learners with the closest to the real world. In the traditional education model, the students' knowledge acquisition is mainly through the experience world and the language and writing world. However, the corresponding education model cannot always combine the two well. Instead, the phenomenon of separation between theory and practice often occurs. Today, the virtual reality world created by information technology is becoming an important bridge to communicate the first two worlds. [4] Web3d has the characteristics of immersion, interactive, and imagination.

(1). The telepresence user feels immersed in the virtual environment presented in the browser.

(2). Multi-sensory users can perceive information in various forms such as visual and auditory.

(3). Interactivity. Users can use natural input and output devices to operate and receive feedback from objects or scenes in a virtual environment with a natural approach.

(4). Authenticity. The movement of objects in a virtual environment is close to the laws of physics.

(5). The establishment and display of three-dimensional space are in a high-efficiency virtual environment which does not depend on the client's hardware performance and can be rendered in real time. The amount of data to be transmitted is small and can be streamed.

\section{Web3D Applications}

(1). Chemistry and physics experiment teaching introduction and exhibition of expensive experimental instruments in chemistry and physics, and visits to experimental spaces that are impossible to enter, such as nuclear reactors, particle collision space, etc., are not a difficult task for Web3D. Of course, for primary and secondary school teaching, the main application of Web3D is to display microscopic objects such as molecular models. We can use Cult3D to make models of chemical molecules, so that students can more intuitively feel the size and relative position of atoms in chemical molecules. At the same time, we can see through the data modification that the work efficiency of using Web3D will be greater than the placement of the physical model.

(2). The use of geography and archeology to visit museums you cannot reach in the world and to study private collections of paintings or sculptures that have never been opened to the public. At the same time, the use of Web3D technology can also enable real treasures to be protected, while also satisfying people's desire to contact world treasures. Museums such as the Forbidden City began a 
virtual tour of the Internet.

(3). The outlook of learning outcomes and experimental results If Web3D technology is applied to the learning process of architectural design and home improvement design, learners can use virtual reality technology to see the results of the finished product design at the beginning of design, and learn and modify the results. In the course of biological experiments, waiting for the growth of plants or the growth of animals is a time-consuming task. If we are eager to see the results, we can use Web3D virtual reality to achieve satisfactory results. Web3D technology has a very powerful role in the application of teaching environment. Reasonable application of Web3D technology can enhance the overall effect of teaching[5].

\section{Conclusion}

As a new thing, the construction of a virtual experimental environment is receiving universal attention. The construction of our country's virtual experimental environment is still in its infancy. There is still a lack of interaction, neglect of the guiding role of teachers, and lack of control over student learning. There are still difficulties and obstacles in the development of Web3 D technology. This is mainly manifested in two aspects: (1) There is no uniform standard. Each Web3 D technology is a solution developed by different companies, and they all use different formats and methods. There is no uniform standard, and the implementation of 3D on the Web will take some time. (2) Plug-in problems. Almost every company develops Web3D technology standards that require the support of their own plug-ins. These plug-ins come in hundreds of ways. From k to a few megabytes, this will inevitably limit the enthusiasm of some people under conditions of unfavorable bandwidth. Although there are still many problems in various technologies, it is accompanied by the popularity of the Internet, network technologies and hardware. With the rapid development of facilities and the increase of network bandwidth, the three-dimensional network will become the mainstream of future network multimedia development. Web3 D will no longer be far away.

\section{Acknowledgements}

This work was supported by 2017 Jiangxi Provincial Education and Teaching Reform Project (JXJG-17-22-16,JXJG-17-22-6), 2017 Jiangxi Provincial Educational Science Planning Project (17YB276).

\section{References}

[1] Tang Shiliang. Research and Implementation of Virtual Experiment System Based on Web3D[D]. Beijing University of Posts and Telecommunications, 2011.

[2] Jiang Yanping, Xia Wangsheng, Huang Xinyuan. Comparison of Several Web3D Technologies[J]. Journal of North China University of Technology, 2003(01):24-28.

[3] Li Xiaotao, Tang Shiliang, Wan Diwen. Development of Web-based Virtual Experiment Environment Using Web3D Technology[J]. Journal of Beijing Radio \& TV University, 2007(1):49-52.

[4] Xu Fei, Li Jing. Application Research of Web 3D Technology in Teaching Environment[J]. Science \& Technology Information (Academic Research), 2008(12): 348-349.

[5] Lu Guohui. Interior Design Method Based on 3D Technology and Its Application[D]. Xiamen University, 2013. 